\title{
A new class of plane symmetric solution
}

\author{
Hongsheng Zhang* \\ Korea Astronomy and Space Science Institute, Daejeon 305-348, Korea and \\ Department of Astronomy, Beijing Normal University, Beijing 100875, China \\ Hyerim $\mathrm{Noh}^{\dagger}$ \\ Korea Astronomy and Space Science Institute, Daejeon 305-348, Korea \\ Zong-Hong Zhu $\mathrm{Z}^{\ddagger}$ \\ Department of Astronomy, Beijing Normal University, Beijing 100875, China
}

(Dated: September 20, 2021)

\begin{abstract}
A new class of static plane symmetric solution of Einstein field equation generated by a perfect fluid source is put forward. A special family of this new solution is investigated in detail. The constraints on the parameters by different energy conditions are studied. The classical stability of this solution is discussed. The junction conditions matching to Minkowski metric and Taub metric are analyzed respectively.
\end{abstract}

PACS numbers: 04.20.Jb, 04.20.Cv, 04.20.-q

\section{INTRODUCTION}

Though considerable amounts of vacuum solutions of Einstein equation are known, but, frankly speaking, the physical interpretation of many of them remains unsettled [1]. As emphasized in [2], the key to physical interpretation is to find out the nature of the sources which generate these vacuum spaces. Singularity theorem is one of the most important and profound theorem in classical general relativity. For a deeper understanding and control of the singularities, the physical characters, such as mass densities, internal pressures, of the sources are imperative. For example in black hole solutions, where only singularity "generates" the whole space, investigating of how a matter distribution gives rise to the black hole space is necessary to judge the physical significance (or lack of it) of the complete analytic extensions of these solutions.

Concerning how we obtain a comprehending of the sources, there is really no alternative for constructing an interior solution for a matter distribution which matches to the vacuum space in question. We will present a new class of plane symmetric solution sourced by perfect fluid. One may be curious about where we can find a realistic plane symmetric space. In the researches of the early universe, people find there may be several-time phase transitions when the universe is cooled down, and many topological defects, such as monopole, cosmic string, and domain wall may survive today. Theoretically, equivalence principle, as principle of general relativity, says that a gravity field can be simulated by an accelerating effect. For the case of a uniformly accelerating observer, an exact simulation should be plane symmetric (but curved) space.

The static vacuum plane symmetric solution, Taub solution was obtained more than 50 years ago [3]. The solution is asymptotically flat but there is a serious time-like singularity in Taub space. People believe that the singularity should be superseded by some matter source, for some attempts to seek the sources, see [7]. Taub solution permits a three-dimensional Euclidean symmetry group, but it is not homogeneous. Here a solution is called homogeneous if it permits three translational space-like Killing vectors. Taub solution only permits two: It is not homogeneous in $z$ direction. Further, it is shown that generally speaking any singularity free source with reflective symmetry for plane symmetric vacuum space must violate dominant energy condition [4].

In electromagnetics the electronic field of an infinite homogeneously charged flat board is homogeneous. By analogy, in linearized gravity theory the solution for a $\delta$-function like source has been derived [5], which is spatially homogeneous and seems to contradict with [4]. It is suggested that the counterpart of the Einstein theory to the gravitational field of a massive Newtonian plane should be described by the Rindler solution rather than Taub's [ $[6]$.

We will present a new class of plane symmetric solution with perfect fluid source, which definitely violates the dominant energy condition. We study some various properties of this new solution and do some preliminary studies on how to glue it to a vacuum space.

\footnotetext{
* Electronic address: hongsheng@kasi.re.kr

$\dagger$ Electronic address: hr@kasi.re.kr

$\ddagger$ Electronic address: zhuzh@bnu.edu.cn
} 
This paper is organized as follow: In the next section we will present our solution. Because the energy momentum tensor is very miry and hence the physical properties of this solution are difficult to analyse, the full form of the energy momentum tensor is casted into the appendix. We then give a special class of the solution as a starting point of the further discussions. In section III we study the constraint on the parameters by the different energy conditions. In section IV, we investigate the stability property of this solution. In section $\mathrm{V}$, we try to glue this sourced solution to vacuum solutions. The cases of Minkowski and Taub are discussed respectively. A summary and some discussions are presented in section VI.

\section{THE SOLUTION}

We find that the following plane symmetric metric

$$
d s^{2}=-f(z)^{2} d t^{2}+d z^{2}+e^{2 a z} f(z)^{2} e^{-2\left[a z+\frac{h(z) \arctan \left(e^{a z} \sqrt{\frac{w}{c}}\right)}{\sqrt{w c}}-d h(z)\right]}\left(d x^{2}+d y^{2}\right),
$$

solves the Einstein field equation with a perfect fluid source. Here $a, w, c, d$ are 4 real parameters, and

$$
\begin{gathered}
f(z)=c e^{-a z}+w e^{a z}, \\
h(z)=-c e^{-a z}+w e^{a z} .
\end{gathered}
$$

Clearly there are four Killing fields $\frac{\partial}{\partial t}, \frac{\partial}{\partial x}, \frac{\partial}{\partial y}$ and $-y \frac{\partial}{\partial x}+x \frac{\partial}{\partial y}$. The latter three span a Euclidean group $G_{3}$, which implies the plane symmetry. The metric (1) solves the Einstein field equation with a source in perfect fluid form

$$
T=(\rho(z)+p(z)) U \otimes U+p(z) g,
$$

where $T$ denotes the energy momentum tensor of the fluid, $U$ stands for four-velocity of the fluid and $g$ denotes the metric tensor. Since the exact forms of $p(z)$ and $\rho(z)$ are very involved, we just present them in the appendix. The energy momentum tensor of metric (10) is too complicated to make further analysis. Alternatively we may concentrate on a special family of solution (1).

First, we set $w=0$, and hence (1) reduces to

$$
d s^{2}=-c^{2} e^{-2 a z} d t^{2}+d z^{2}+c^{2} e^{-2\left(a z-1+d c e^{-a z}\right)}\left(d x^{2}+d y^{2}\right),
$$

where we have used

$$
\lim _{w \rightarrow 0} \frac{h(z) \arctan \left(e^{a z} \sqrt{\frac{w}{c}}\right)}{\sqrt{w c}}=-1 .
$$

Then, we rescale the coordinates $t, x$ and $y$ by

$$
c t \rightarrow t, \quad e c x \rightarrow x, \quad \text { ecy } \rightarrow y .
$$

So (5) further reduces to

$$
d s^{2}=-e^{-2 a z} d t^{2}+d z^{2}+e^{-2\left(a z+d e^{-a z}\right)}\left(d x^{2}+d y^{2}\right)
$$

Introducing new variables

$$
a^{\prime}=-a, \quad b^{\prime}=-d,
$$

and for convenience labeling $a^{\prime}$ by $a$ and $b^{\prime}$ by $b$, we derive a special solution as follow,

$$
d s^{2}=-e^{2 a z} d t^{2}+d z^{2}+e^{2\left(a z+b e^{a z}\right)}\left(d x^{2}+d y^{2}\right) .
$$

There are two free parameters in the above solution. With the above metric $\rho(z)$ and $p(z)$, respectively given by (74), (75) for the original metric (11), are reduced to extraordinarily simple form

$$
\begin{aligned}
& \rho(z)=-a^{2}\left(3+8 b e^{a z}+3 b^{2} e^{2 a z}\right), \\
& p(z)=a^{2}\left(3+4 b e^{a z}+b^{2} e^{2 a z}\right),
\end{aligned}
$$

where we have set $8 \pi G \equiv 1$, and $G$, as usual, denotes the Newton gravitational constant. In this article we will concentrate on metric (10). Before studying the detailed properties of (10), we first investigate the various limitations of it. Obviously when $a=0$ (10) degenerates to Minkowski metric. When $b=0$, it becomes

$$
d s^{2}=d z^{2}+e^{2 a z}\left(-d t^{2}+d x^{2}+d y^{2}\right),
$$

which is just anti-de Sitter (AdS) metric in an unattractive coordinate system. Surely it describes a homogeneous and isotropic solution in all directions, not only a plane symmetric one. 


\section{ENERGY CONDITIONS}

More and more cosmological evidences imply that exotic matters violating energy conditions, such as weak, strong, dominant or null energy conditions, do exist in our universe. And the partition of the exotic matter takes a much more value than that of dust matter. But different requirements on the energy-momentum tensor have been used to prove several pivotal theorems, for example singularity theorem and positive energy theorem, in classical general relativity. It may be helpful to investigate the properties of the matter in the source by different energy conditions. Further, it is pointed out that the source of Taub must violate the dominant energy condition if the source is reflective symmetric and without spacetime singularity [4]. Now we begin to study the parameter regions permitted by weak, strong and dominant energy conditions. Note that weak and strong energy conditions are two independent conditions. In this section we consider a plane source with finite thickness, which resides in the region $z \geq 0$. Our solution is an interior solution of plane source, thus the pressure $p(z)$ should vanish naturally at some distance from the "ground", $z=z_{0}$, that is, we should match it to a vacuum solution. This condition implies

$$
p\left(z_{0}\right)=a^{2}\left(3+4 b e^{a z_{0}}+b^{2} e^{2 a z_{0}}\right)=0 .
$$

The two roots of the above equation reads

$$
\begin{gathered}
b_{1}=-e^{-a z_{0}}, \\
b_{2}=-3 e^{-a z_{0}} .
\end{gathered}
$$

$b_{1}$ and $b_{2}$ denote the two branches of the solution which have finite thickness. We call them "little branch" and "large branch", respectively.

To show the physical meanings of the parameters, we calculate the mass per area of this slab $\alpha$.

$$
\alpha=\int_{0}^{z_{0}} d z e^{2\left[a z+b e^{a z}\right]} \rho(z),
$$

where $\rho(z)$ is defined in (11). For the little branch $b=-e^{-a z_{0}}$, the integral yields,

$$
\alpha=\frac{a}{8} e^{-a z_{0}}\left[-5 e^{-2+3 a z_{0}}+e^{-2 e^{-a z_{0}}}\left(14 e^{a z_{0}}+2 e^{2 a z_{0}}+e^{3 a z_{0}}-12\right)\right] .
$$

For the large branch, the result is

$$
\alpha=\frac{a}{72} e^{-a z_{0}}\left[191 e^{-6+3 a z_{0}}+e^{-2 e^{-a z_{0}}}\left(126 e^{a z_{0}}+6 e^{2 a z_{0}}+e^{3 a z_{0}}-324\right)\right] .
$$

We see that the mass per area is proportional to $a$, so it can be treated as a mass parameter of the slab. Whether the stuff filled in the slab is ordinary or exotic in some sense depends on the value of $a z_{0}$. Different energy conditions impose different constraints on $a z_{0}$.

Weak energy condition (WEC) requires that the energy momentum tensor of the source $T$ satisfies,

$$
T(Z, Z) \geq 0
$$

for any time-like vector $Z$, which equals

$$
\begin{gathered}
\rho=-a^{2}\left(3+8 b e^{a z}+3 b^{2} e^{2 a z}\right) \geq 0, \\
\rho+p=-a^{2}\left(4 b e^{a z}+2 b^{2} e^{2 a z}\right) \geq 0 .
\end{gathered}
$$

First, we consider the little branch $-e^{-a z_{0}}$. We then replace $b$ by $-e^{-a z_{0}}$ in (21) and (22). For further discussions we investigate three cases depending on the sign of $a$.

Case I: $a>0$. Inequality (21) requires

$$
e^{a z_{0}} \leq \frac{3}{4-\sqrt{7}}
$$

and inequality $(22)$ is satisfied spontaneously in this settlement.

Case II: $a<0$. Inequality (21) requires

$$
e^{a z_{0}} \geq \frac{3}{4+\sqrt{7}}
$$


Inequality (22) requires

$$
e^{a z_{0}} \geq 1 / 2
$$

therefore the permitted range of $a$ and $z_{0}$ is

$$
e^{a z_{0}} \geq 1 / 2
$$

The case $a=0$ degenerates to Minkowski space, which is a trivial case satisfying any energy conditions. In the interval,

$$
e^{a z_{0}} \in\left[1 / 2, \frac{3}{4-\sqrt{7}}\right],
$$

WEC always can be satisfied for any real $a$.

Second, we consider the large branch $b=-3 e^{-a z_{0}}$. Mimicking the above discussions for the case of little branch we find that WEC can not be satisfied for any $a$ and $z_{0}$.

Then we turn to the strong energy condition (SEC), which requires

$$
\operatorname{Ric}(Z, Z) \geq 0
$$

where Ric denotes the Ricci tensor of metric (10), and $Z$ is an arbitrary time-like vector. The condition (28) equals to

$$
\begin{array}{r}
\rho+p=-a^{2}\left(4 b e^{a z}+2 b^{2} e^{2 a z}\right) \geq 0, \\
\rho+3 p=a^{2}\left(6+4 b e^{a z}\right) \geq 0 .
\end{array}
$$

First, similarly, we consider the little branch $b=-e^{-a z_{0}}$. Also, we investigate the three cases depending on the sign of $a$, respectively.

Case I: $a>0$. The inequality (29) has been discussed in the case WEC, which is satisfied naturally. One finds (30) is also satisfied naturally. So SEC imposes no constraint on the parameters in this case.

Case II: $a<0$. Inequality (29) has been analyzed before, which requires

$$
e^{a z_{0}} \geq 1 / 2
$$

and inequality (30) requires

$$
e^{a z_{0}} \geq 2 / 3
$$

The case $a=0$ degenerates to Minkowski space, as we have pointed out, which is a trivial case marginally satisfying any energy conditions. For arbitrary $a$ the requirement of SEC always can be satisfied in the interval,

$$
e^{a z_{0}} \in[2 / 3, \infty)
$$

Second, we consider the branch $b=-3 e^{-a z_{0}}$ for SEC. Mimicking the above discussions we find that SEC can not be satisfied for any $a$ and $z_{0}$. We see the case $b=-3 e^{-a z_{0}}$ may violate both WEC and SEC for any values of parameters $a$ and $z_{0}$.

The dominant energy condition (DEC) requires

$$
\rho \geq|p|
$$

For removing the calculation of absolute value, we consider the cases of $p<0$ and $p \geq 0$, respectively. First, $p<0$ requires that

$$
\left(b+e^{-a z_{0}}\right)\left(b+3 e^{-a z_{0}}\right)<0,
$$

whose solution is an empty set. Second, for the case $p \geq 0$, DEC requires

$$
3+6 b e^{a z}+2 b^{2} e^{2 a z} \leq 0
$$

which does not have non-empty solution set either. Hence we conclude that this solution always violates DEC. Recalling that [4] demonstrates that any reasonable source of Taub solution must violate DEC, one sees that our present solution just satisfies this requirement. 


\section{STABILITY}

In this section we give an initiative discussion on the global stability of the solution. The basic idea of our investigation is best presented by considering an analogy. A Newtonian celestial body, for instance, the Globe, is stable, which can be understood as follow: If the Globe undergoes an small perturbation, expanding or contracting a little, the total energy (including gravitational energy and the energy of the particles of which the Globe consists) of new configuration is higher than the original configuration. Hence the Globe is stable in view of least energy principle.

A similar argument can be applied to our solution. The first problem is the energy of the gravitational field. People recognized that there is no covariant energy momentum of gravitational field long ago. Different expressions correspond to different boundary conditions in the Hamiltonian approach of quasi-local mass [8]. Here we adopt the Landau-Lifschitz expression [9]. According to Landau-Lifschitz expression, the energy momentum of gravitational field reads

$$
\begin{aligned}
S^{\mu \nu}=\frac{1}{2}[ & \left(2 \Gamma_{\beta \gamma}^{\alpha} \Gamma_{\alpha \rho}^{\rho}-\Gamma_{\beta \rho}^{\alpha} \Gamma_{\gamma \alpha}^{\rho}-\Gamma_{\beta \alpha}^{\alpha} \Gamma_{\gamma \rho}^{\rho}\right)\left(g^{\mu \beta} g^{\nu \gamma}-g^{\mu \nu} g^{\beta \gamma}\right)+g^{\mu \beta} g^{\gamma \alpha}\left(\Gamma_{\beta \rho}^{\nu} \Gamma_{\gamma \alpha}^{\rho}+\Gamma_{\gamma \alpha}^{\nu} \Gamma_{\beta \rho}^{\rho}-\Gamma_{\alpha \rho}^{\nu} \Gamma_{\beta \gamma}^{\rho}-\Gamma_{\beta \gamma}^{\nu} \Gamma_{\alpha \rho}^{\rho}\right) \\
& \left.+g^{\nu \beta} g^{\gamma \alpha}\left(\Gamma_{\beta \rho}^{\mu} \Gamma_{\gamma \alpha}^{\rho}+\Gamma_{\gamma \alpha}^{\mu} \Gamma_{\beta \rho}^{\rho}-\Gamma_{\alpha \rho}^{\mu} \Gamma_{\beta \gamma}^{\rho}-\Gamma_{\beta \gamma}^{\mu} \Gamma_{\alpha \rho}^{\rho}\right)+g^{\beta \gamma} g^{\alpha \rho}\left(\Gamma_{\beta \alpha}^{\mu} \Gamma_{\gamma \rho}^{\nu}-\Gamma_{\beta \gamma}^{\mu} \Gamma_{\alpha \rho}^{\nu}\right)\right],
\end{aligned}
$$

where $\Gamma$ denotes the affine connection of metric (10) and $g_{\mu \nu}$ denotes the component of (10). The non-zero components of $\Gamma$ are

$$
\begin{array}{r}
\Gamma_{t z}^{t}=\Gamma_{z t}^{t}=a, \quad \Gamma_{t t}^{z}=a e^{2 a z}, \quad \Gamma_{x x}^{z}=-a g_{x x}, \quad \Gamma_{y y}^{z}=-a g_{x x}, \\
\Gamma_{z x}^{x}=\Gamma_{x z}^{x}=a\left(1+b e^{a z}\right), \quad \Gamma_{y z}^{y}=\Gamma_{z y}^{y}=a\left(1+b e^{a z}\right) .
\end{array}
$$

Substituting (38) into (37) we derive the energy density of gravity,

$$
\rho_{\text {gra }}=-S_{0}^{0}=a^{2}\left(4+4 b e^{a z}\right) .
$$

Then, in the little branch the density per area of gravity is

$$
\beta=\int_{0}^{z_{0}} d z e^{2\left[a z-e^{a\left(z-z_{0}\right)}\right]} \rho_{\text {gra }} .
$$

Performing the calculation directly, we obtain [11]

$$
\beta=2 a\left(e^{-2+2 a z_{0}}-e^{-2 e^{-a z_{0}}}\right),
$$

which is also proportional to $a$. For the large branch, the area density becomes

$$
\beta=\int_{0}^{z_{0}} d z e^{2\left[a z-3 e^{a\left(z-z_{0}\right)}\right]} \rho_{\mathrm{gra}}
$$

which yields,

$$
\beta=2 a\left(e^{-6+2 a z_{0}}-e^{-6 e^{-a z_{0}}}\right) .
$$

The total mass associated with the unit slab is $\sigma=\alpha+\beta$. In the little branch, by using (18) and (41),

$$
\begin{aligned}
\sigma & =\alpha+\beta \\
& =\frac{a}{8} e^{-a z_{0}}\left[11 e^{-2+3 a z_{0}}+e^{-2 e^{-a z_{0}}}\left(-2 e^{a z_{0}}+2 e^{2 a z_{0}}+e^{3 a z_{0}}-12\right)\right] .
\end{aligned}
$$

Imitating the case of a Newtonian star, we impose a perturbation to $z_{0}$,

$$
\delta \sigma=\frac{a^{2}}{4} e^{-2-2 e^{-a z_{0}}-2 a z_{0}}\left(-12 e^{2}+4 e^{2+a z_{0}}+2 e^{2+2 a z_{0}}+2 e^{2+3 a z_{0}}+e^{2+4 a z_{0}}+11 e^{2 e^{-a z_{0}}+4 a z_{0}}\right) \delta z_{0} .
$$

Numerical calculations show that stagnation points dwell at $z_{0}=-0.292 a^{-1}$ and $z_{0}=-1.245 a^{-1}$. Further we find

$$
\begin{gathered}
\left.\delta^{2} \sigma\right|_{r_{0}=-0.292 a^{-1}}<0, \\
\left.\delta^{2} \sigma\right|_{r_{0}=-1.245 a^{-1}}>0,
\end{gathered}
$$


where we have used $a<0$, since $z_{0}>0$. We conclude that the configuration described by (10) is stable when the parameters $a, r_{0}$ are constrained by $a r_{0}=-1.245$. The resulting stable metric is a one parameter family,

$$
d s_{\text {sta }}^{2}=-e^{2 a z} d t^{2}+d z^{2}+e^{2\left(a z-3.473 e^{a z}\right)}\left(d x^{2}+d y^{2}\right) .
$$

In the large branch, similar to the little branch, the total mass of a unit slab reads,

$$
\begin{aligned}
\sigma & =\alpha+\beta \\
& =\frac{a}{72} e^{-a z_{0}}\left[335 e^{-6+3 a z_{0}}+e^{-6 e^{-a z_{0}}}\left(-18 e^{a z_{0}}+6 e^{2 a z_{0}}+e^{3 a z_{0}}-324\right)\right] .
\end{aligned}
$$

Imposing a perturbation to $z_{0}$, we reach

$$
\delta \sigma=\frac{a^{2}}{36} e^{-6-6 e^{-a z_{0}}-2 a z_{0}}\left(-972 e^{6}+108 e^{6+a z_{0}}+18 e^{6+2 a z_{0}}+6 e^{6+3 a z_{0}}+e^{6+4 a z_{0}}+335 e^{6 e^{-a z_{0}}+4 a z_{0}}\right) \delta z_{0} .
$$

Numerical calculations show that two stagnation points for $\delta \sigma=0$ inhabit at $z_{0}=-0.3163 a^{-1}$ and $z_{0}=0.9770 a^{-1}$. Further, one can show

$$
\begin{aligned}
\left.\delta^{2} \sigma\right|_{r_{0}=-0.3163 a^{-1}} & >0, \\
\left.\delta^{2} \sigma\right|_{r_{0}=0.9770 a^{-1}} & >0,
\end{aligned}
$$

therefore, both of the two stagnation points are stable. They correspond to two spaces of one parameter family,

$$
\begin{gathered}
d s_{\mathrm{sta} 1}^{2}=-e^{2 a z} d t^{2}+d z^{2}+e^{2\left(a z-4.116 e^{a z}\right)}\left(d x^{2}+d y^{2}\right), \\
d s_{\mathrm{sta} 2}^{2}=-e^{2 a z} d t^{2}+d z^{2}+e^{2\left(a z-1.13 e^{a z}\right)}\left(d x^{2}+d y^{2}\right),
\end{gathered}
$$

respectively.

\section{MATCHING TO VACUUM SOLUTIONS}

Surely, (1) and (10) are rigorous solutions with perfect fluid sources, which can be filled in the whole space. However, there is a true singularity if interval of $z$ is not confined. In fact, the Ricci scalar $R$ reads,

$$
R=-2 a^{2}\left(6+10 b e^{a z}+3 b^{2} e^{2 a z}\right) .
$$

When $z$ goes to $\infty(-\infty)$, Ricci scalar will be divergent for a positive (negative) $a$. We hereby consider the case that this solution is only valid in a finite region and the spacetime is vacuum out of this region.

The gravitational field must satisfy two boundary conditions: 1 . The metric is continuous across the boundary surface, and 2. The extrinsic curvatures measured by the different sides of the boundary surface relate to each other by

$$
[K-h \operatorname{tr}(K)]^{ \pm}=-\tau,
$$

in which $K$ denotes the extrinsic curvature of the boundary, $h=g-\frac{\partial}{\partial z} \otimes \frac{\partial}{\partial z}$ represents the induced metric on the boundary, $\tau$ is the energy-momentum tensor confined to the boundary, and [ $]^{ \pm}$denotes the jump at the boundary, i.e., for a quantity $Q,[Q]^{ \pm}=\lim _{\left(z-z_{0}\right) \rightarrow 0^{+}} Q(z)-\lim _{\left(z-z_{0}\right) \rightarrow 0^{-}} Q(z)$. The exterior vacuum space has to be plane symmetric to match to metric (10). First we study the most simple case that the vacuum out of the source region is Minkowskian geometry. Then we analyse the junction condition matching to the well known non-flat plane symmetric space: (static) Taub space.

Before discussing the boundary condition between the slab and the vacuum, we impose a $Z_{2}$ (reflective) boundary condition at $z=0$. The continuity condition is naturally satisfied and the jump condition generates

$$
[K-h \operatorname{tr}(K)]^{0+}=-\frac{1}{2}(\tau)^{0},
$$

where $0+$ labels the value of a quantity at $z=0$ going from the positive direction, and 0 denotes the value of a quantity at $z=0$. Using the above equation we derive

$$
\tau_{\mu}^{\nu}=2 \operatorname{diag}(2 a(1+b), a(2+b), a(2+b))
$$


where $b=-e^{-a z_{0}}$ or $b=-3 e^{-a z_{0}}$, depending on the little or large branch. Now we write the Minkowskian metric in the following chart,

$$
d s_{\text {Min }}^{2}=-m^{2} d t^{2}+d z^{2}+n^{2}\left(d x^{2}+d y^{2}\right)
$$

where $m$ and $n$ are positive constants. The continuous condition yields

$$
\begin{aligned}
m & =e^{a z_{0}}, \\
n & =e^{a z_{0}+b e^{a z_{0}}},
\end{aligned}
$$

which corresponds to "time contraction" and "length contraction", though $m, n$, may be greater than 1 so that they represent "time dilation" and "length dilation". From the jump condition (56) we obtain $\tau$ in induced chart by (59) in the little branch,

$$
\tau_{\mu}^{\nu}=\operatorname{diag}(0,-a,-a)
$$

while in the large branch,

$$
\tau_{\mu}^{\nu}=\operatorname{diag}(4 a, a, a)
$$

$\tau$ vanishes when $a=0$, which is consistent with our expectation because the interior metric degenerates to Minkowski when $a=0$.

Next we discuss the conditions matching to the Taub metric. The Taub's metric reads,

$$
d s^{2}=-z^{-2 / 3} k^{2} d t^{2}+d z^{2}+z^{4 / 3} l^{2}\left(d x^{2}+d y^{2}\right),
$$

where $k, l$ are two positive constants. The continuous condition requires

$$
3 a z_{0}=1+2 \ln k l,
$$

in the little branch, and

$$
a z_{0}=1+\frac{2}{3} \ln k l
$$

in the large branch.

The jump condition (56) gives, in the induced chart by (64) for the little branch

$$
\tau_{\mu}^{\nu}=\operatorname{diag}\left(-\frac{8}{3} z_{0}^{-5 / 3}, \frac{2}{3} z_{0}^{-1}+a-\frac{4}{3} z_{0}^{-5 / 3}, \frac{2}{3} z_{0}^{-1}+a-\frac{4}{3} z_{0}^{-5 / 3}\right),
$$

where $z_{0}$ is given by (65). And for the large branch

$$
\tau_{\mu}^{\nu}=\left(a+\frac{1}{3} z_{0}^{-1}\right) \operatorname{diag}(-4,-1,-1)
$$

where $z_{0}$ takes the value in (66). If we require the matching is perfect, that is, boundary energy momentum vanishes, we arrive at $a z_{0}=-1 / 3$.

On the other hand, if perfect matching condition is imposed in the little branch, one needs an infinitely thick slab, which can be derived from (67).

\section{CONCLUSIONS AND DISCUSSIONS}

In this article we present a global plane symmetric solution of Einstein field equation with a perfect fluid source, which we interpret as the source of some plane symmetric vacuum space. Four Killing vectors, including a time like Killing vector, are permitted in this solution. We find a chart in which the metric is written in time coordinate orthogonal form. Then we give a special and much simpler class of the original solution as the starting point for further investigations.

We find the ranges of the parameters in which WEC and SEC can be satisfied, respectively. Interestingly, we find that DEC is always violated, no matter what values the parameters are taken. 
We study the stability properties of this solution. For the little branch, the stable point dwells at $z_{0}=-1.245 a^{-1}$, and for the large branch, the stable points inhabit at $z_{0}=-0.3163 a^{-1}$ and $z_{0}=0.9770 a^{-1}$.

We do some primary researches on matching to vacuum solutions. Minkowski's and Taub's are studied respectively. Furthermore, for clarifying the physical contents of this solution, we should study the weak field approximation to find some thing like Newton force among test particles.

The general form of the plane symmetric solution (1) with a perfect fluid source must imply more rich physical and mathematical properties. It may deserve to study further in the future. It should be noted that in the present article we have examined only the classical stability under $z$-direction perturbations, though it is the most important perturbations of plane symmetric space. The other forms of classical perturbations, quantum and semi-classical perturbations should be thoroughly investigated further.

Acknowledgments. H.Noh was supported by grant No. C00022 from the Korea Research Foundation. Z.H. Zhu was supported by the National Natural Science Foundation of China, under Grant No. 10533010, by Program for New Century Excellent Talents in University (NCET) and SRF for ROCS, SEM of China.

\section{APPENDIX}

One derives the Einstein tensor of metric (11), whose components read,

$$
\begin{aligned}
& G_{t}^{t}=\left[-16 b^{3} c^{4} d u^{3}+16 b^{4} c^{3} d u^{5}+12 b^{3} c^{4} d u^{3}+45 b^{2} c^{4} u^{2} v^{2}+3 b^{7} d^{2} c u^{10}+12 b^{7 / 2} c^{5 / 2} u^{5} v+18 b^{2} c^{6} d^{2}-\right. \\
& 12 b^{4} c^{3} d u^{5}+45 b^{3} c^{5} d^{2} u^{2}+18 b^{6} c^{2} d^{2} u^{8}+45 b^{5} c^{3} d^{2} u^{6}+18 b c^{5} v^{2}-6 b^{6} \sqrt{b c} d u^{10} v+18 b^{9 / 2} c^{3 / 2} u^{7} v- \\
& 16 b^{2} c^{4} e^{2 a r}-120(b c)^{7 / 2} d u^{4} v+6 b^{5} c u^{8}+6 b^{11 / 2} c^{1 / 2} u^{9} v-24 b^{2} c^{5} d u-8 b c^{6} / u+24 b^{3 / 2} c^{9 / 2} v+ \\
& 8 \sqrt{b c} c^{5} u^{-1} v-12 b^{5 / 2} c^{7 / 2} u^{3} v+45 b^{4} c^{2} u^{6} v^{2}+8 b^{4} c^{2} u^{6}+24 b^{5} c^{2} d u^{7}+60 b^{4} c^{4} d^{2} u^{4}-90 b^{9 / 2} c^{5 / 2} d v+ \\
& b^{5 / 2} c^{9 / 2} d u+8 b^{6} c d u^{9}-8 b c^{5}-6 \sqrt{b c} c^{6} u^{-2} v+18 b^{5} c u^{8} v^{2}+3 c^{6} u^{-2} v^{2}+6 b c^{5}-16 b^{2} c^{3} u^{4}+ \\
& 3 b c^{7} d^{2} u^{-2}-18 b^{3 / 2} c^{9 / 2} u v-6 b^{1 / 2} c^{11 / 2} u^{-1} v-8 b^{5} c u^{8}+4 b^{3} c^{3} u^{4}-18 b^{5} c^{2} d u^{7}+6 b c^{6} d / u- \\
& 16 b^{4} c^{2} u^{6}+3 b^{6} u^{10} v^{2}-36 b^{3 / 2} c^{11 / 2} v+16 b^{2} c^{3} \sqrt{b c} u^{3} v-16 b^{4} c^{3} u^{5} v-90 b^{2} c^{4} \sqrt{b c} d u^{2} v-24 b^{5} c^{2} u^{7} v+ \\
& \left.+8 b^{2} c^{4} u^{2}-36 b^{5} c \sqrt{b c} d u^{8} v-8 b^{5} \sqrt{b c} v+60 b^{3} c^{3} u^{4} v^{2}-6 b^{6} c d u^{9}\right] a^{2}(b c)^{-1}\left(c+b u^{2}\right)^{-4}, \\
& G_{z}^{z}=-\left[4 b c^{6} d u^{-1}-6 b^{2} c^{6} d^{2}-2 b^{5} \sqrt{b c} u^{7} v+40 b^{3} c^{3} \sqrt{b c} d v / u-8 b^{3} c^{3} u^{2}+2 \sqrt{b c} c^{6} u^{-2} v-6 b^{2} c^{5} d / u-\right. \\
& 4 b c^{5}+6 b^{5} c^{2} d u^{5}-15 b^{4} c^{2} u^{4} v^{2}-8 b^{4} c^{3} d u^{3}-12 b^{5} c^{2} d u^{5}-6 b^{4} c \sqrt{b} c u^{5} v-4 b^{5} c u^{6}+\sqrt{b c} c^{5} v u^{-3}+ \\
& 2 b^{6} c d u^{7}+2 b^{6} \sqrt{b c} d u^{8} v+12 b^{4} c \sqrt{b c} u^{5} v+12 b^{5} c \sqrt{b c} u^{6} v+8 b^{3} c^{4} d u-15 b^{2} c^{4} v^{2}-6 b^{6} c^{2} d^{2} u^{6}- \\
& 6 b c^{5} u^{-2} v^{2}-b^{6} u^{8} v^{2}-4 b^{6} c d u^{7}+30 b^{2} c^{4} \sqrt{b c} d v+4 b^{4} c^{3} d u^{3}+8 b^{3} c^{3} u^{2}+4 b^{5} c u^{6}+6 b c^{4} \sqrt{b c} v / u+ \\
& 12 b c^{5} \sqrt{b c} u^{-2} v-15 b^{3} c^{5} d^{2}+30 b^{4} c^{2} \sqrt{b c} u^{4} v+8 b^{3} c^{2} \sqrt{b c} u^{3} v+4 b c^{5} \sqrt{b c} u^{-2}-2 b c^{6} \sqrt{b c} d u^{-3}- \\
& c^{6} u^{-4} v^{2}-b^{7} c d^{2} u^{8}-4 b^{3} c^{4} d u+4 b^{5} \sqrt{b c} u^{7} v+12 b^{2} c^{5} d / u+4 b^{2} c^{3} \sqrt{b c} u v-b c^{7} d^{2} u^{-4}-12 b c^{4} \sqrt{b c} v / u+ \\
& \left.8 b^{2} c^{4}-20 b^{4} c^{4} d^{2} u^{2}-20 b^{3} c^{3} u^{2} v^{2}-8 b^{3} c^{3} \sqrt{b c} u v-4 c^{5} \sqrt{b c} u^{-3} v-6 b^{5} c u^{6} v^{2}\right] a^{2}(b c)^{-1}\left(c+b u^{2}\right)^{-4},
\end{aligned}
$$

where $u=e^{a z}, v=\arctan \left(u \sqrt{\frac{b}{c}}\right)$ and

$$
G_{x}^{x}=G_{y}^{y}=G_{z}^{z}
$$

The Einstein tensor is checked by software Maple. We see that, though the Einstein tensor is rather messy, it reveals a significant property of metric (1): it describes a solution of plane symmetric sourced by perfect fluid, whose energy momentum is in the form (4). Then in coordinate system (11),

$$
\begin{array}{r}
T_{t}^{t}=G_{t}^{t}, \\
T_{x}^{x}=T_{y}^{y}=T_{z}^{z}=G_{z}^{z} .
\end{array}
$$

We use the convention $8 \pi G \equiv 1$ throughout this article. So, in the comoving system of the perfect fluid, $U=\partial / \partial t$,

$$
\begin{gathered}
\rho=-T_{t}^{t}, \\
p=T_{z}^{z} .
\end{gathered}
$$


[1] H. Stephani, D. Kramer, C. Hoenselaers, E. Herlt, M. A MacCallum, Exact Solutions of Einstein's Field Equations , Cambridge University Press, 2003.

[2] W. B. Bonnor, M. A. P. Martins, Class. Quantum Grav. 8 (1991) 727.

[3] A.H. Taub, Ann.Math. 53 (1951) 472.

[4] A.D. Dolgov, and I.B. Khriplovich, Gen.Rel.Grav. 21 (1989) 13.

[5] A. Vilenkin, Phys. Rev. D 23, 852 (1981).

[6] M. F. A. da Silva, A. z. A. Wang and N. O. Santos, Phys. Lett. A 244, 462 (1998) arXiv:gr-qc/9706071.

[7] J. Horsky and J. Novotny, J.Phys.A 2 (1969) 251; J. Ipser and P. Sikivie, Phys.Rev.D 30 (1984) 712; O,Gron and H.H. Soleng, Phys.Lett.A 165 (1992) 191; B.W. Bonnor, Gen. Rel. Grav. 24, 551 (1992); B. Jensen and J. Kucera, Phys. Lett. A195, 111 (1994); M.L. Bedran, M.O. Calvao, F.M. Paiva and I.D. Soares, Phys. Rev. D55, 3431 (1997); A F F Teixeira, gr-qc/0502013.

[8] C. M. Chen and J. M. Nester, Grav. Cosmol. 6, 257 (2000) arXiv:gr-qc/0001088, and references therein.

[9] L. D. Landau, E. M. Lifschitz, The Classical Theory of Fields 4th edn, Pergamon Press Ltd, 1975.

[10] J. D. Brown and J. W. York, Phys. Rev. D 47, 1407 (1993).

[11] However, we should keep alert on this result since it is well known uncertainty of the gravitational energy density. The quasi-local energy defined in [10] equals the value of the Hamiltonian that generates unit time translations orthogonal to a spacelike hypersurface $D$ at its boundary two-surface $\partial D$. It seems a reasonable definition of the quasi-local energy for gravity because of this property. Now, in our case, $D$ is a unit-square thick slab, and naturally, $\partial D$ is composed of the four walls and two covers of it. The 3-boundary is defined as the product of $\partial D$ with timelike world lines orthogonal to $D$ at $\partial D$. The induced 3-metric on the 3-boundary can be written as

$$
\gamma=-e^{2 a z} d t^{2}+d z^{2}+e^{2\left(a z+b e^{a z}\right)} d x^{2}
$$

on the walls along $x$ direction,

on the walls along $y$ direction, and

$$
\begin{gathered}
\gamma=-e^{2 a z} d t^{2}+d z^{2}+e^{2\left(a z+b e^{a z}\right)} d y^{2}, \\
\gamma=-e^{2 a z} d t^{2}+e^{2\left(a z+b e^{a z}\right)}\left(d x^{2}+d y^{2}\right),
\end{gathered}
$$

on the two covers, respectively. Note that the induced metric is not well defined on the joint lines gluing the slab and the two covers and the jointing lines between the walls. Hereby the boundary energy momentum tensor is not well defined there. Just as well the six lines are zero-measure sets, and then we can omit the energy associated with it without changing the result. With this construction it is easy to show that the quasi-local gravitational energy enclosed by the slab and is exactly the same as the result by using Landau-Lifschitz expression for our settlement. 